\title{
Understanding "fouling" in extremely complex petroleum mixtures
}

\author{
Aikaterini Kondyli, Wolfgang Schrader* \\ Max-Planck-Institut für Kohlenforschung, Kaiser-Wilhelm-Platz 1, D-45470 Mülheim/Ruhr, Germany
}

\section{MATERIALS AND METHODS}

\section{Simulation of fouling under laboratory conditions}

A lab build reactor was used for fouling simulation. The experimental set up was the following: A quartz crucible was filled with $0.3 \mathrm{~mL}$ of a gas condensate and was placed inside a stainless steel autoclave. Afterwards, a quartz cover was placed on the top of the crucible in order to avoid sample losses. The autoclave was then positioned in a heating plate which was operated using a temperature controller. The sample was heated for 3 days at a temperature of $150{ }^{\circ} \mathrm{C}$, $250{ }^{\circ} \mathrm{C}, 350{ }^{\circ} \mathrm{C}$ or $450{ }^{\circ} \mathrm{C}$ under both air (OC) or argon (IC) atmosphere. In order to verify the mechanism, the model nitrogen compound of quinoline in the presence of oct-3-ene as initiator was dissolved in $0.1 \mathrm{~mL}$ of toluene and heated for 3 days at $150{ }^{\circ} \mathrm{C}$ and $450{ }^{\circ} \mathrm{C}$.

\section{Sample preparation for MS analysis}

All samples were first dissolved in toluene (HPLC grade, Sigma-Aldrich, Germany) and then diluted with methanol (LC-MS grade, J. T. Baker, Germany) (1:4 v/v) to a final concentration of $250 \mathrm{ppm}$ and then analyzed without further treatment.

\section{Instruments and methods}

For mass spectrometric analysis a research-type Orbitrap Elite (Thermo Fisher, Bremen, Germany) was used. ${ }^{19,} 30$ Mass spectra were recorded in a range of $\mathrm{m} / \mathrm{z}, 150-1000$ using spectral stitching method (scanning the whole mass range in windows of 30 a with 5 a overlap) at a resolving power of $R=480,000$ and 960,000 (FWHM at $\mathrm{m} / z$ 400). Ionization was performed using an electrospray (ESI) source in positive mode with a stainless steel needle at a capillary voltage of $4 \mathrm{kV}$. Nebulization was assisted by a sheath gas flow of 3.0 a.u. (arbitrary units) while the auxiliary and the sweep gas flow were set at 0.0 a.u. The transfer capillary temperature was set at $275{ }^{\circ} \mathrm{C}$. For the fragmentation experiments MS/MS measurements were performed by collision-induced dissociation (CID). The precursor mass was selected with an isolation window of $1.0 \mathrm{a}$ and the isolated ions were kinetically excited with high (around $45 \%$ ) energy using helium as collision gas.

\section{Data analysis}

External mass calibration was performed prior to sample analysis in order to meet a mass accuracy of $<1 \mathrm{ppm}$. The recorded mass spectra were recombined by Xcalibur 4.0 software (Thermo Fisher, Bremen, Germany) and further processed using the Composer software (version 1.5.0, Sierra Analytics, Modesto, CA, USA). Calculations and peak assignments were carried out using the following restrictions regarding the number of possible elements and the number of double bond equivalent: H 0-1000, C: 0-200, N: 0-3, O: 0-6, S: 0-2 and DBE: 0-100. The calculated molecular formulae were sorted into heteroatom classes based on their denoted Kendrick mass defects and their double bond equivalent distribution. The obtained mass lists were transferred to Excel (Microsoft Office Professional Plus 2010, Microsoft Corporation, Redmond, WA, USA) for further data evaluation. Double bond equivalent refers to the sum of the rings and double bonds present within a molecule and is calculated from the general $\mathrm{C}_{\mathrm{c}} \mathrm{H}_{\mathrm{h}} \mathrm{N}_{\mathrm{n}} \mathrm{O}_{\mathrm{o}} \mathrm{S}_{\mathrm{s}}$ of the analyte using the following equation: DBE $=\mathrm{c}-\frac{\mathrm{h}}{2}+\frac{\mathrm{n}}{2}+1$. A higher DBE value indicates higher aromaticity of a compound.

\section{Electron Microscopy}

Electron microscopic measurments were obtained by using a powder preparation technique on a Hitachi HF-2000 $200 \mathrm{kV}$ coldFEG TEM. 